\title{
Putting a dampener on inflammation
}

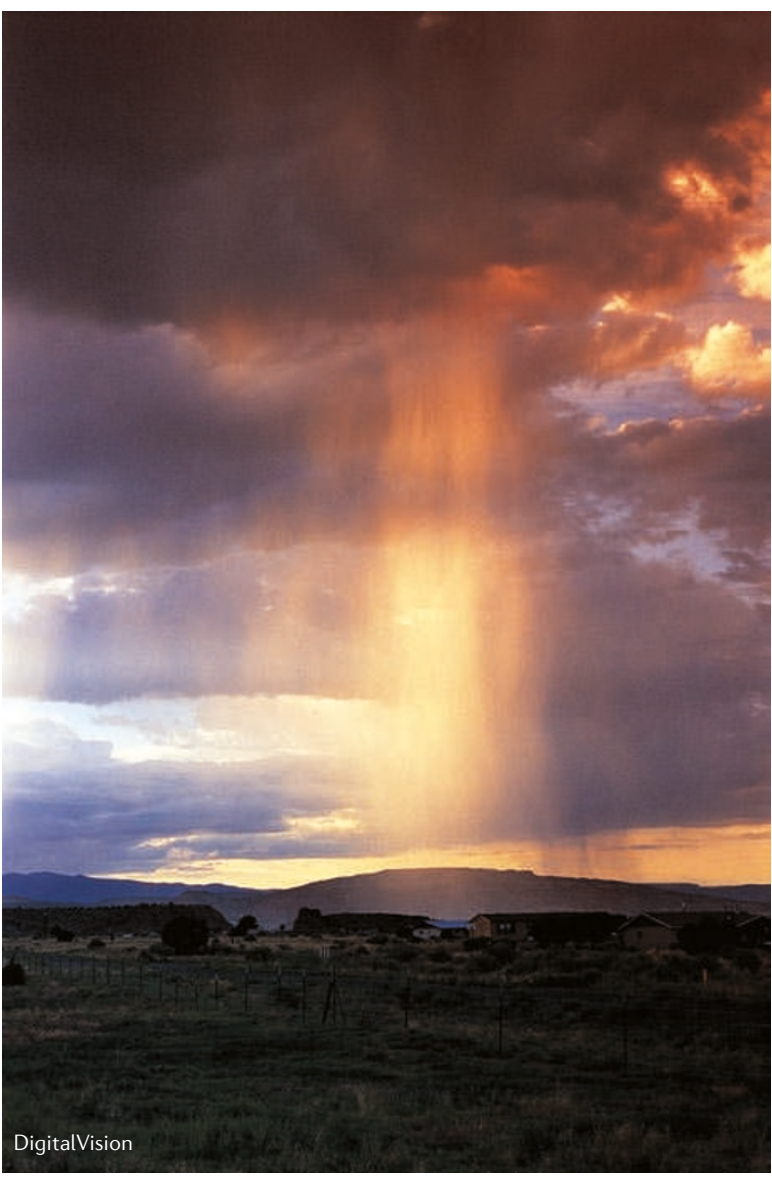

Multiple sclerosis is a chronic, demyelinating disease of the CNS that is thought to have an inflammatory aetiology. High levels of glutamate are found in the brains of patients with multiple sclerosis, but the link between this excitatory neurotransmitter and the immunopathology is not clear. Now, Fallarino et al. suggest a role for metabotropic glutamate receptor 4 (mGluR4) signalling in dampening the immune response during neuroinflammation, highlighting a pathway that could be exploited therapeutically.

Previous work in a rodent model of multiple sclerosis - experimental autoimmune encephalomyelitis (EAE) - had shown that activation of specific mGluRs, including mGluR4, could help to mediate recovery from disease. The authors therefore assessed the clinical symptoms of mice with EAE that lacked the gene encoding mGluR4 ( $\mathrm{Grm} 4^{-/-}$ mice) and discovered that they had more severe symptoms and an earlier onset of disease than wild-type mice.

mRNA profiling of $\mathrm{CD} 4^{+} \mathrm{T}$ cells, key effector immune cells, from the brain and lymph nodes of $\mathrm{Grm}^{-/-}$ mice indicated that differentiation of these cells into pro-inflammatory $\mathrm{T}$ helper $17\left(\mathrm{~T}_{\mathrm{H}} 17\right)$ cells was favoured over differentiation into regulatory $\mathrm{T}$ $\left(\mathrm{T}_{\mathrm{Reg}}\right)$ cells, which was in contrast to wild-type mice. Furthermore, EAEinduced inflammation was greater in the knockout mice, with higher levels of pro-inflammatory cytokines and lower levels of inflammationsuppressing cytokines than in wild-type mice with EAE.

Reverse transcription PCR on various cell types from wild-type mice showed that mGluR4 expression was highest in $\mathrm{CD}^{+} \mathrm{T}$ cells and dendritic cells - immune cells that can promote $\mathrm{T}$ cell differentiation into a regulatory or an inflammatory phenotype depending on signals from the local environment. Cultures of activated T cells from $\mathrm{Grm}^{-/-}$mice had the same cytokine profile and rate of proliferation as those from wild-type mice. By contrast, activated dendritic cells from $\mathrm{Grm}^{-/-}$mice, but not wild-type mice, had a proinflammatory cytokine profile. In particular, these cells secreted high levels of interleukin-6 (IL-6) and low levels of IL-27, a profile that is known to promote the differentiation of naive $T$ cells into $T_{H} 17$ rather than $\mathrm{T}_{\text {Reg }}$ cells. Therefore, the effect of mGluR4 signalling on the T cell phenotype seems to be mediated through dendritic cells.

The intracellular signalling pathway that links dendritic cell activation with the production of $\mathrm{T}_{\mathrm{H}} 17$-type cytokines involves increased cyclic AMP formation. Administration of a positive allosteric modulator of mGluR4 known as PHCCC attenuated this cAMP increase in activated wild-type dendritic cells. In vivo, administration of PHCCC to mice with EAE attenuated disease in a statistically significant, dose-dependent manner and was associated with an increase in $\mathrm{T}_{\text {Reg }}$ cells in the lymph nodes. Thus, allosteric modulators of mGluR4, which are currently under investigation for Parkinson's disease, might provide an opportunity to harness what seems to be a protective effect of glutamate in patients with multiple sclerosis.

ORIGINAL RESEARCH PAPER Fallarino, F. et al.

Metabotropic glutamate receptor-4

modulates adaptive immunity and restrains neuroinflammation. Nature Med. 25 Jul 2010 (doi:10.1038/nm.2183) 